\title{
Separation of Photoabsorption and Compton Scattering Contributions to He Single and Double Ionization
}

\author{
L. Spielberger, ${ }^{1, *}$ O. Jagutzki, ${ }^{1}$ R. Dörner, ${ }^{1}$ J. Ullrich, ${ }^{2}$ U. Meyer, ${ }^{1}$ V. Mergel, ${ }^{1}$ M. Unverzagt, ${ }^{1}$ M. Damrau, ${ }^{1}$ T. Vogt, ${ }^{1}$ \\ I. Ali, ${ }^{1}$ Kh. Khayyat, ${ }^{1}$ D. Bahr, ${ }^{3}$ H. G. Schmidt, ${ }^{3}$ R. Frahm, ${ }^{3}$ and H. Schmidt-Böcking ${ }^{1}$ \\ ${ }^{1}$ Institut für Kernphysik, Universität Frankfurt, D60486 Frankfurt am Main, Germany \\ ${ }^{2}$ GSI, D64220 Darmstadt, Germany \\ ${ }^{3}$ HASYLAB am DESY, D22603 Hamburg, Germany
}

(Received 20 January 1995)

\begin{abstract}
We have experimentally separated the contributions of photoabsorption and Compton scattering to $\mathrm{He}$ single and double ionization for high-energy photon impact by measuring the full momentum vector of the recoiling $\mathrm{He}^{1+, 2+}$ ions. For recoil ions following photoabsorption large momenta and a distinct dipole emission pattern are observed. The ions produced by Compton scattering show small momenta. For the ratio of double to single ionization we find $(1.22 \pm 0.06) \%$ at $8.8_{-1.65}^{+1.5} \mathrm{keV}$ for Compton scattering and $(1.72 \pm 0.12) \%$ at $7.0_{-1.6}^{+2.1} \mathrm{keV}$ for photoabsorption. We compare our data with recent theories.
\end{abstract}

PACS numbers: $32.80 . \mathrm{Fb}, 31.15 .-\mathrm{p}, 32.80 . \mathrm{Dz}$

Compton scattering and photoabsorption are the two fundamental ionizing interactions of a photon with an atom. For the Compton process the momentum and energy transfer of the photon correspond to the final energy and momentum of the emitted electron, whereas for photoabsorption a second particle in the final statethe recoiling target ion-is needed to compensate for the momentum of the free electron. Both interactions are described by single particle operators. Thus double ionization of a $\mathrm{He}$ atom is always mediated by electronelectron correlation. Determining the ratio of double to single ionization $\left(R=\sigma^{2+} / \sigma^{1+}\right)$ by Compton scattering $\left(R_{\mathrm{C}}\right)$ and by photoabsorption $\left(R_{\mathrm{ph}}\right)$, because of the intrinsic differences between the two processes, is one of the most challenging tests of our understanding of these electronelectron correlation effects [1].

In this Letter we demonstrate an experimental approach to distinguish both mechanisms by measuring the recoil momentum of the ions simultaneously with their charge state at a photon energy of about $8 \mathrm{keV}$. Since ions from Compton events and photoionization are distinct in momentum space, this method provides for the first time a separate determination of the ratios $R_{\mathrm{C}}$ and $R_{\mathrm{ph}}$.

Most experimental studies on He double photoionization concentrated on low and intermediate energies [2]. Only in the last few years have experiments in the $\mathrm{keV}$ regime become feasible [3-5] due to novel synchrotron radiation sources. These experiments were aiming to establish the asymptotic high-energy value for $R_{\mathrm{ph}}$. However, as brought to attention by Samson, Green, and Bartlett [6], all those experiments did not allow one to distinguish between Compton scattering and photoabsorption. At about $6 \mathrm{keV}$ the single ionization cross sections for both processes are equal [6-10]. The photoionization cross section scales with $E^{-7 / 2}[11,12]$ in this high-energy regime, whereas the Compton cross section is slightly increasing with energy
[7-9]. Therefore, instead of solving the old problem to settle an experimental value for the high-energy limit of $R_{\mathrm{ph}}$, new questions were added: Is there any difference between $R_{\mathrm{C}}$ and $R_{\mathrm{ph}}$, and what are their values?

From the theoretical side agreement has been achieved [1] in predicting the asymptotic value for $R_{\mathrm{ph}}$ to be $1.67 \%$ [7,12-14], although the predictions fluctuated between $0.32 \%$ [15] and $2.33 \%$ [16] over many years and an experimental proof is still missing. For Compton scattering neither the high-energy limit of $R_{\mathrm{C}}$ nor the energy dependence of $R_{\mathrm{C}}$ before reaching the asymptotic value is clear: Surić et al. [10] calculated $0.8 \%$ approached from below, Andersson and Burgdörfer [1] come to the same asymptotic value but approached from above, and Amusia and Mikhailov [17] predict $1.7 \%$. Thus at $8 \mathrm{keV}$, where the present experiments were performed, the predictions differ by more than a factor of 2 between $0.6 \%$ [10], $1.3 \%$ [1], and $1.65 \%$ from Hino, Bergstrom, and Macek [9].

In order to determine $R_{\mathrm{ph}}$ and $R_{\mathrm{C}}$ separately, not only the charge state but also the momentum of the recoil ions was measured in the present experiment using COLTRIMS (cold target recoil ion momentum spectroscopy) [18-20]. A supersonic He gas jet provides a dense, localized, and internally cold He target. The He gas expands through a $30 \mu \mathrm{m}$ nozzle from a gas reservoir at a pressure of 30 bar into the first chamber. Through a skimmer of $0.6 \mathrm{~mm}$ diam the gas jet enters into the scattering chamber. This setup yields a target of $3 \mathrm{~mm}$ diam and a density of $2 \times 10^{12} / \mathrm{cm}^{2}$. The gas jet atoms have a well-defined offset momentum of 6 a.u. in the $y$ direction resulting from the supersonic expansion. The recoil ions created at the intersection volume with the photon beam are accelerated by a well-defined homogeneous electric field of $96 \mathrm{~V} / \mathrm{cm}$ (see Fig. 1). After a $3 \mathrm{~cm}$ acceleration length the ions pass a $6 \mathrm{~cm}$ field free drift tube and are then postaccelerated to $2000 \mathrm{~V} /$ charge onto a two-dimensional position-sensitive 


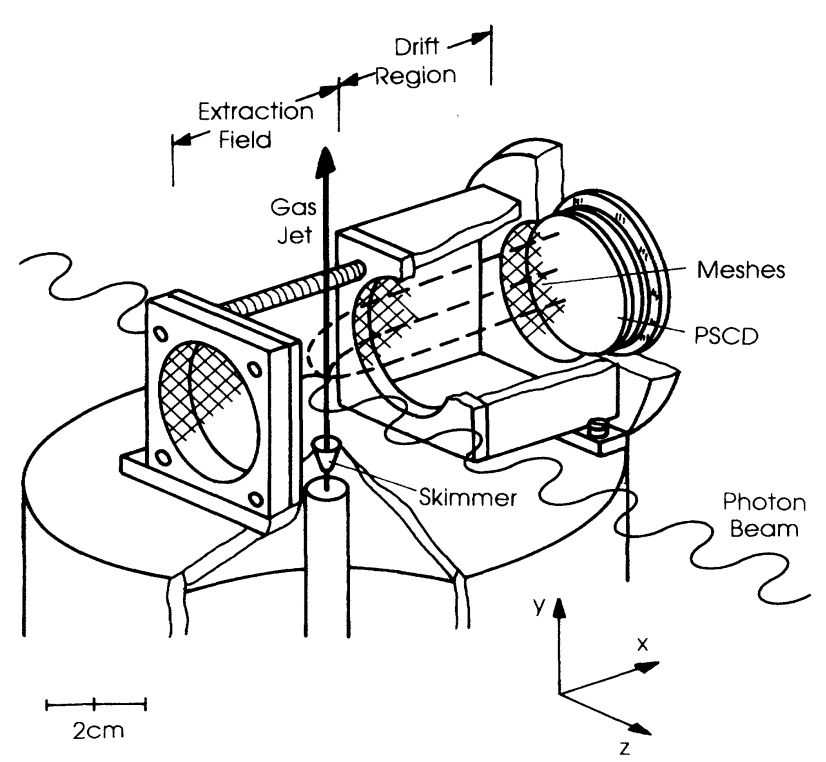

FIG. 1. Recoil-ion momentum spectrometer with supersonic He gas jet. The recoil ions have been measured in coincidence with the beam pulse, the channel-plate detector (PSCD) is twodimensional position sensitive with wedge and strip readout. The electric field vector of the linear polarized light is parallel to the extraction field.

chevron-channel-plate detector of $40 \mathrm{~mm}$ active diam with wedge and strip readout. The electrical extraction field is sufficiently strong to project all created ions onto the detector. The ions are detected in coincidence with the timing signal of the beam pulse provided from the storage ring. From the time of flight (TOF) we obtain the recoil ion charge state and the momentum component parallel to the field direction. The two momentum components perpendicular to the extraction field are calculated from the position on the channel-plate detector (see Fig. 1) and the TOF.

The experiment has been performed at the undulator beamline BW1 of HASYLAB at DESY in Hamburg. Two experiments have been carried out at the same time at this beamline. The main experiment used a small band pass of $x$ rays reflected out of the direct beam by a Be single crystal monochromator of $0.25 \mathrm{~mm}$ thickness operated in Laue transmission geometry. Our experiment was performed with all photons passing in a forward

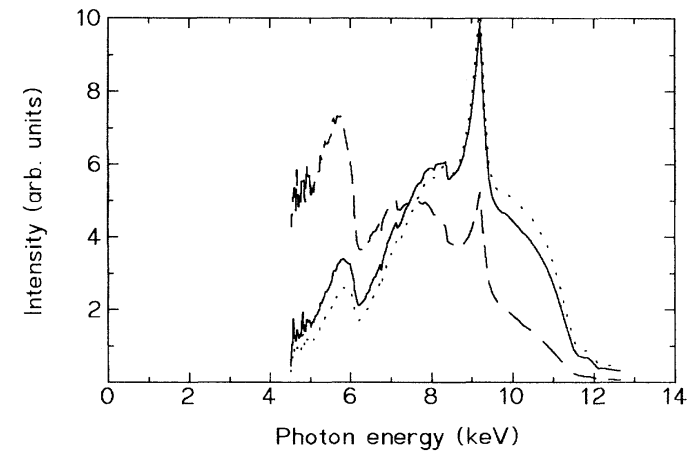

FIG. 2. Full line: photon energy distribution as used for the experiment. Dashed line: photon energy distribution folded with the $E^{-7 / 2}$ dependence of the $\mathrm{He}^{1+}$ photoionization cross section [11,12]; dotted line: photon energy distribution folded with calculated Compton scattering cross sections [7-9].

direction through the $\mathrm{Be}$ crystal. Thus the radiation used in this experiment was not monoenergetic. The photon spectrum is shown in Fig. 2. It was measured after the experiment with a $\mathrm{Si}$ (111) single crystal in $\Theta-2 \Theta$ geometry. The spectrum is dominated by the third harmonic of the undulator, which was set to $9.2 \mathrm{keV}$. It is cut below $5 \mathrm{keV}$ by the absorption of the beamline windows (carbon, beryllium, and aluminum) and the $\mathrm{Be}$ crystal, and on the high-energy side at about $11 \mathrm{keV}$ by two Au coated mirrors. These mirrors were used to focus the light in the vertical and horizontal directions. A beamspot of less than $1 \times 2 \mathrm{~mm}$ was achieved by two sets of adjustable slits about 1 and $1.5 \mathrm{~m}$ upstream of the collision region. The experiment was performed at a flux of about $1 \times 10^{14}$ photons/sec over the whole spectrum yielding about $80 \mathrm{He}^{1+}$ ions/sec.

The measured momentum distributions of the $\mathrm{He}^{1+}$ and $\mathrm{He}^{2+}$ ions are shown in Figs. 3(a)-3(c). Figure 3(a) is plotted in the $p_{x}-p_{y}$ plane, where $x$ is the direction of the electric field vector of the linear polarized light and $y$ is that of the He gas jet. For the geometry, see Fig. 1. The data of Figs. 3(a)-3(c) are summed over momenta in the $z$ direction, -30 a.u. $<p_{z}<30$ a.u. Because of the rotational symmetry of the system, the distribution must be symmetrical with respect to the $p_{x}$ axis. Residual $\mathrm{He}$ gas atoms can be seen in Fig. 3(a) at $p_{y}=-6$ a.u. From energy and momentum conservation we obtain for photoabsorption and Compton scattering

$$
\begin{array}{ll}
\vec{p}_{\gamma}+\vec{p}_{\mathrm{He}}=\vec{p}_{\mathrm{He}^{1+}}+\vec{p}_{e} & \left(\text { photo, } \mathrm{He}^{1+}\right), \\
\vec{p}_{\gamma}+\vec{p}_{\mathrm{He}}=\vec{p}_{\mathrm{He}^{2+}}+\vec{p}_{e 1}+\vec{p}_{e 2} & \left(\text { photo, } \mathrm{He}^{2+}\right), \\
\vec{p}_{\gamma}+\vec{p}_{\mathrm{He}}=\vec{p}_{\gamma^{\prime}}+\vec{p}_{\mathrm{He}^{1+}}+\vec{p}_{e} & \left(\text { Compton, } \mathrm{He}^{1+}\right), \\
\vec{p}_{\gamma}+\vec{p}_{\mathrm{He}}=\vec{p}_{\gamma^{\prime}}+\vec{p}_{\mathrm{He}^{2+}}+\vec{p}_{e 1}+\vec{p}_{e 2} & \left(\text { Compton, } \mathrm{He}^{2+}\right) .
\end{array}
$$

The left hand side gives the momentum in the initial state. The incoming photon momentum $\vec{p}_{\gamma}$ is 2.4 a.u. at $9 \mathrm{keV}$ and $\vec{p}_{\mathrm{He}}$, the momentum of the He atom as a whole, is approximately zero $(<0.2$ a.u., resulting from the internal He gas jet temperature). $\vec{p}_{\gamma^{\prime}}, \vec{p}_{e_{i}}$, and $\vec{p}_{\mathrm{He}^{1+2+}}$ are the final-state momenta of the scattered photon, the emitted electrons, and the recoil ion. 


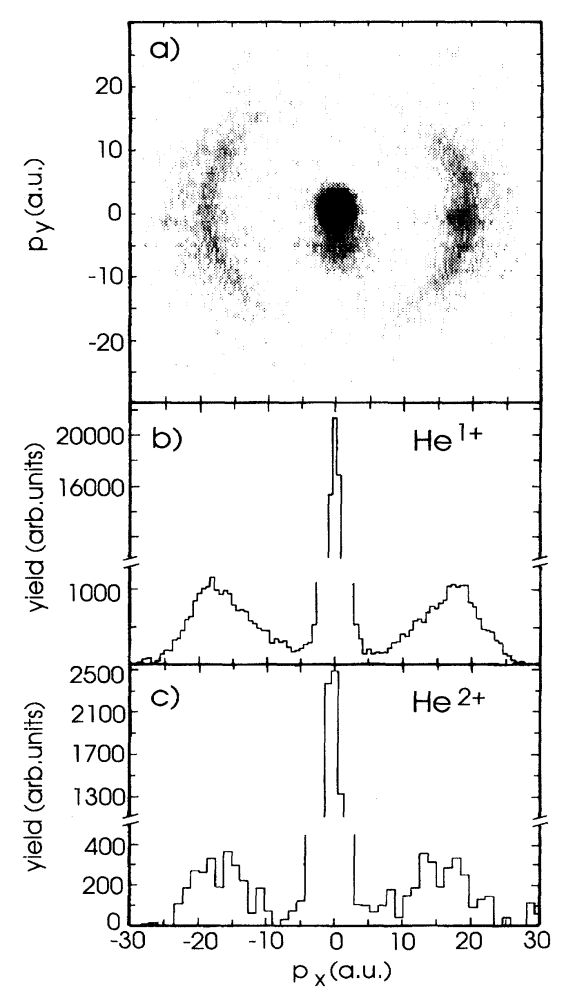

FIG. 3. (a) Measured momentum distribution of $\mathrm{He}^{1+}$ ions in the $x-y$ plane, integrated over the momentum in the $z$ direction between -30 and 30 a.u. (b) Projection of (a) on the $x$ direction. (c) Same as (b) for the $\mathrm{He}^{2+}$ charge state. The statistical fluctuations result from background subtraction.

The $\mathrm{He}^{1+}$ and $\mathrm{He}^{2+}$ momentum distributions [Figs. 3(a)-3(c)] show a dominant contribution close to zero momentum resulting from Compton scattering. For the Compton process the nucleus is a spectator to first order. The momentum change $\left(\vec{p}_{\gamma^{\prime}}-\vec{p}_{\gamma}\right)$ of the photon is transferred to the ejected electron(s) leaving the nucleus behind with its initial momentum. The nucleus compensates the initial momentum distribution of the target electrons (Compton profile). Since the He atoms are in a well-defined initial state of zero momentum, one expects for Compton scattering small recoil ion momenta on the order of 1 a.u., as can be seen from our experiment. This is similar to what we have found recently for electron impact [19] and proton impact [21]. Samson et al. have recently used this fact to measure the cross section for $\mathrm{He}^{1+}$ production by Compton scattering [5]. In contrast, for single ionization by photoabsorption the recoil ion has a fixed momentum for each photon energy, since the ion must compensate the sum of initial photon and final electron momentum. For $9 \mathrm{keV}$ photon impact the recoil ions are distributed on a sphere in momentum space of 25.7 a.u. radius. With respect to the laboratory frame this sphere has to be shifted by $\vec{p}_{\gamma}=2.4$ a.u. in the beam direction due to the initial photon momentum. On this sphere the recoil ions have a density distribution mainly given by a dipole emission pattern due to the linear polarization of the synchrotron radiation. From theoretical investigations $[16,22]$ only minor contributions of less than $2 \%$ from higher order transitions than the dipole portion are expected for this experiment. The dominant dipole pattern can be seen nicely in Fig. 3(a). The width of the momentum distribution is mainly due to the energy spread of the incoming photons. The dashed line in Fig. 2 represents the photon energy distribution folded with the $E^{-7 / 2}$ dependence of the photoabsorption cross section $[11,12]$. After integrating over the full momentum sphere twice as many $\mathrm{He}^{1+}$ ions from Compton scattering as from photoabsorption are observed in the experiment. This is in good agreement with the result calculated on the basis of the photon energy distribution and the calculated absolute cross sections for photoabsorption $\left(\sigma_{\mathrm{ph}}^{1+}\right)$ and Compton scattering $\left(\sigma_{\mathrm{C}}^{1+}\right)[7-9]$.

The $\mathrm{He}^{2+}$ momentum distribution is found to be very similar to the one for single ionization. This is in agreement with previous findings that at this high energy the dominant $\mathrm{He}^{2+}$ production process for photoabsorption is the emission of one very fast and one slow electron [16].

By integrating over the respective volumes in momentum space we obtain $R_{\mathrm{C}}$ and $R_{\mathrm{ph}}$ separately. The results are $(1.22 \pm 0.06) \%$ at $8.8_{-1.65}^{+1.5} \mathrm{keV}$ for Compton scattering and $(1.72 \pm 0.12) \%$ at $7.0_{-1.6}^{+2.1} \mathrm{keV}$ for photoabsorption (see Fig. 4). The respective energy is the mean value of the folded photon spectrum (see Fig. 2). The horizontal error bars indicate the spectral region from which $67 \%$ of all $\mathrm{He}^{1+}$ ions originate. Because of the high photon flux, the statistical error for $R_{\mathrm{C}}$ is negligible $(0.01 \%)$. By our technique we are also able to definitely rule out the three major systematic errors of all former high-energy studies, which are small contributions from low-energy stray light, ionization by secondary electrons, and charge exchange of the ions with the target gas $[3,4]$. Lower energetic light would cause photoabsorption and would yield smaller recoil ion momenta. Secondary electrons would not be restricted to the path of the photon beam and therefore result in He ion creation along the gas jet and not only at the intersection point with the photon beam, which can be separated from the detector position. The well-localized gas jet and the good background pressure of $2 \times 10^{-7} \mathrm{mbar}$ prevent secondary collisions of the ions resulting in charge exchange. Thus the only remaining systematic error could be a charge state dependence of the detection efficiency of the channel plate. This was checked in two ways. First the amplification of the channel plate was reduced by a factor of 2 (the pulse height of the channel-plate signal is recorded for each event) by reducing the overall voltage by $100 \mathrm{~V}$. Second, measurements were performed with two different postacceleration voltages of 2000 and $1000 \mathrm{~V}$. Both tests yielded the same $R_{\mathrm{C}}$ within $5 \%$. The 


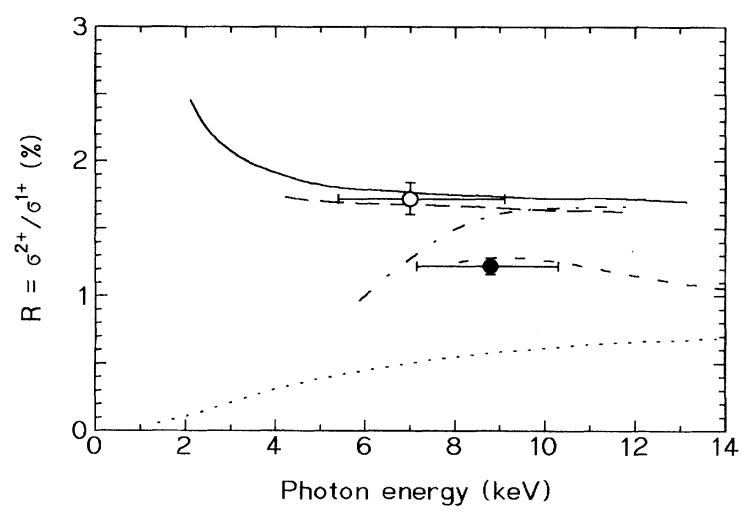

FIG. 4. Ratios of double to single ionization. Full circle: experimental ratio for Compton scattering $\left(R_{\mathrm{C}}\right)$; open circle: experimental ratio for photoabsorption $\left(R_{\mathrm{ph}}\right)$. The data have been measured with light of an energy distribution as given in Fig. 2. The horizontal error bars indicate the energy region from which $67 \%$ of all $\mathrm{He}^{1+}$ ions result (based on cross sections from [7-9,11]. Full line: $R_{\mathrm{ph}}$ from Andersson and Burgdörfer [7]; broken line: $R_{\mathrm{ph}}$ from Hino et al. [9]; dashed line: $R_{\mathrm{C}}$ from Andersson and Burgdörfer [1]; dotted line: $R_{\mathrm{C}}$ from Surić et al. [10]; dash-dotted line: $R_{\mathrm{C}}$ from Hino et al. [9].

given error of $R_{\mathrm{C} / \mathrm{ph}}$ is the sum of our estimate of the systematical error based on these cross checks and the statistical error. The one of $R_{\mathrm{ph}}$ is somewhat larger and mainly due to the background subtraction for the $\mathrm{He}^{2+}$ charge state. Ionization of the residual gas was the main contribution for the total recoil ion production; only about $4 \%$ of all events result from helium photoabsorption. These counts are distributed over a large volume in momentum space making background subtraction much more difficult than for the Compton effect.

For photoabsorption our experiment is in excellent agreement with the prediction of $1.67 \%$ by different authors [7,12-14]. For Compton scattering our measured ratio is close to the prediction of Andersson and Burgdörfer, and we can definitely rule out the values of $R_{\mathrm{C}}=0.6 \%$ as predicted by Surić et al. [10] and of $R_{\mathrm{C}}=1.65 \%$ as predicted by Hino, Bergstrom, and Macek [9]. According to theoretical estimates [7] we have already reached the high-energy asymptotic value for $R_{\mathrm{ph}}$. This result is supported by the value $R=(1.6 \pm 0.3) \%$ found by Levin et al. at $2.8 \mathrm{keV}$ which agrees with our ratio. This value represents only photoabsorption, because at this energy the Compton cross section is on the order of $1 \%$ of the one for photoabsorption [7]. For Compton scattering the emitted electron energy is still significantly below $500 \mathrm{eV}$, and we expect to not yet have reached the asymptotic regime. Therefore it cannot yet be determined if $R_{\mathrm{C}}$ and $R_{\mathrm{ph}}$ have the same value in the high-energy limit as predicted by Amusia and Mikhailov [17]. For a further investigation we plan an experiment at about $50 \mathrm{keV}$ to reach the asymptotic regime for Compton scattering.

In conclusion, we have experimentally demonstrated that photoabsorption and Compton scattering yield signifi- cantly distinct recoil ion momentum distributions. We have exploited this difference in momentum space to experimentally separate the processes for single and double ionization of $\mathrm{He}$ at a photon energy of about $8 \mathrm{keV}$. We find a significant difference in the ratio of double to single ionization by photoabsorption and Compton scattering.

The work was financially supported by BMFT, DFG, and the EG. These experiments would not have been possible without the patient acceptance of us by the main synchrotron radiation users, namely, K. Kjaer, G. Brezesinski, L. Leiserowitz, and co-workers. We acknowledge helpful discussion with C.L. Cocke, J. Burgdörfer, P. Bergstrom, T. Surić, M. Amusia, R. Driezler, H. J. Lüdde, and J. R. Samson. We received indispensable help in building the supersonic gas jet from U. Buck. We are grateful for the support of University Frankfurt for the transportation of the experimental setup to DESY, Hamburg, in particular, to E. Suck and Ch. Kazamias.

*Electronic address: spielberger@ikf.uni-frankfurt.de

[1] L. Andersson and J. Burgdörfer, Phys. Rev. A 50, R2810 (1994).

[2] N. Berrah et al., Phys. Rev. A 48, R1733 (1993), and references therein.

[3] J. C. Levin et al., Phys. Rev. Lett. 67, 968 (1991).

[4] J. C. Levin et al., Phys. Rev. A 47, R16 (1993).

[5] J. Samson, Z. He, R. Bartlett, and M. Sagurton, Phys. Rev. Lett. 72, 3329 (1994).

[6] J. Samson, C. Green, and R. Bartlett, Phys. Rev. Lett. 71, 201 (1993).

[7] L. Andersson and J. Burgdörfer, Phys. Rev. Lett. 71, 50 (1993).

[8] P. Bergstrom, Jr., K. Hino, and J. Macek, Phys. Rev. A 51, 3044 (1995).

[9] K. Hino, P. Bergstrom, and J. Macek, Phys. Rev. Lett. 72, 1620 (1994).

[10] T. Surić, K. Pisk, B. Logan, and R. Pratt, Phys. Rev. Lett. 73, 790 (1994).

[11] T. Ichihara, K. Hino, and J. McGuire, Phys. Rev. A 44, R6980 (1991).

[12] A. Dalgarno and H. Sadeghpour, Phys. Rev. A 46, R3591 (1992).

[13] F. Byron and C. Joachain, Phys. Rev. 164, 1 (1967).

[14] T. Åberg, Phys. Rev. A 2, 1726 (1970).

[15] J. Samson, Phys. Rev. Lett. 65, 2863 (1990).

[16] M. Y. Amusia, E. Drukarev, V. Gorshkov, and M. Kazachkov, J. Phys. B 8, 1247 (1975).

[17] M. Y. Amusia and A. Mikhailov, J. Phys. B (to be published).

[18] J. Ullrich et al., Commun. At. Mol. Phys. 30, 285 (1994).

[19] R. Dörner et al., Phys. Rev. Lett. 72, 3166 (1994).

[20] V. Mergel et al., Phys. Rev. Lett. 74, 2200 (1995).

[21] R. Dörner et al., J. Phys. B 28, 435 (1995).

[22] R.H. Pratt and L. LaJohn (private communication). 